Proyecciones Journal of Mathematics

Vol. 35, No 1, pp. 99-117, March 2016.

Universidad Católica del Norte

Antofagasta - Chile

\title{
Coupled lower and upper solution approach for the existence of solutions of nonlinear coupled system with nonlinear coupled boundary conditions
}

\author{
Imran Talib \\ University of Management and Tech., Pakistán \\ Naseer Ahmad Asif \\ University of Management and Tech., Pakistán \\ and \\ Cemil Tunc \\ Yuzuncu Yil University, Turkey \\ Received : November 2015. Accepted : January 2016
}

\begin{abstract}
The present article investigates the existence of solutions of the following nonlinear second order coupled system with nonlinear coupled boundary conditions (CBCs)

$$
\left\{\begin{array}{l}
-u^{\prime \prime}(t)=f_{1}(t, v(t)), \quad t \in[0,1] \\
-v^{\prime \prime}(t)=f_{2}(t, u(t)), \quad t \in[0,1] \\
\mu\left(u(0), v(0), u^{\prime}(0), v^{\prime}(0), u^{\prime}(1), v^{\prime}(1)\right)=(0,0) \\
\nu(u(0), v(0))+(u(1), v(1))=(0,0)
\end{array}\right.
$$

where $f_{1}, f_{2}:[0,1] \times \mathbf{R} \rightarrow \mathbf{R}, \mu: \mathbf{R}^{6} \rightarrow \mathbf{R}^{2}$ and $\nu: \mathbf{R}^{2} \rightarrow \mathbf{R}^{2}$ are continuous functions. The results presented in [7, 11] are extended in our article. Coupled lower and upper solutions, Arzela-Ascoli theorem and Schauder's fixed point theorem play an important role in establishing the arguments. Some examples are taken to ensure the validity of the theoretical results.
\end{abstract}

Keywords : Lower and upper solutions, Nonlinear coupled system, Coupled nonlinear boundary conditions, Arzela-Ascoli theorem, Schauder's fixed point theorem. 


\section{Introduction}

The lower and upper solution methodology has been widely investigated in studying boundary value problems (BVPs) of nonlinear differential equations. The idea was firmly established by the work of Perron [13] on the Dirichlet problem for harmonic functions. In late 1960s, Jackson [12] established the theory for treating BVPs of second order nonlinear ordinary differential equations. Remarkable contributions were also made by Schmitt in [14]. Zhang [15] discussed some notable applications to characterize the existence of positive solutions to the Dirichlet problem of a type of sublinear differential equations. In [16] Zhang established the existence results for the second order nonlinear periodic BVPs and considered the forced pendulum equations with curvature and Duffing type equations as applications.

CBCs appear while studying of Sturm-Liouville problems, reactiondiffusion equations, mathematical biology, chemical systems, and engineering $[17,18,21,22,23]$ and [19] chapter 13. In [20] Leung investigated the applications of $\mathrm{CBCs}$ in mathematical biology by studying the following reaction-diffusion system for prey-predator interaction:

$$
\begin{gathered}
u_{t}(t, x)=\sigma_{1} \Delta u+u(a+f(u, v)), \quad t \geq 0, \quad x \in \Omega \subset \mathbf{R}^{n}, \\
\mathrm{v}_{t}(t, x)=\sigma_{2} \Delta v+v(r+g(u, v)), \quad t \geq 0, \quad x \in \Omega \subset \mathbf{R}^{n},
\end{gathered}
$$

subject to $\mathrm{CBCs}$

$$
\frac{\partial u}{\partial \eta}=0, \quad \frac{\partial v}{\partial \eta}-p(u)-q(v)=0 \quad \text { on } \quad \partial \Omega,
$$

where $f, g: \mathbf{R}^{2} \rightarrow \mathbf{R}$ have Hölder continuous partial derivatives up to second order in compact sets, $\eta$ is a unit outward normal at $\partial \Omega$ and $p$ and $q$ have Hölder continuous first derivatives in compact subsets of $[0, \infty)$. Also, $\Delta=\sum_{i=1}^{n} \frac{\partial^{2}}{\partial x_{i}^{2}}, a, r, \sigma_{1}, \sigma_{2}$ are positive constants. The functions $u(t, x)$ and $v(t, x)$ respectively represent the density of prey and predator at $t \geq 0$ and at position $x=\left(x_{1}, x_{2}, \ldots, x_{n}\right)$. In [18] the same kind of CBCs are studied for biochemical system. Cardanobile and Mugnolo in [22] investigated the following parabolic system with CBCs: 


$$
\frac{\partial u}{\partial t}(t, x)=\mathcal{L} u(t, x), \quad t \geq 0, \quad x \in \Omega \in \mathbf{R}^{n}, f \mid \partial \Omega \in \mathcal{Y}, \quad \frac{\partial f}{\partial v} \in \mathcal{Y}^{\perp},
$$

where $\mathcal{Y}^{\perp}$ is closed subspace of $L^{2}(\partial \Omega ; W)$, the unknown function $u$ takes values in a separable Hilbert space $W$ and $\mathcal{L}$ is an elliptic operator with operator-valued symbol.

The study of ordinary differential systems (ODSs) with coupled and non-coupled boundary conditions has also attracted many authors. The reader can study $[4,5,6,7,24,25]$ and references therein. A second order ordinary differential system (ODS) firstly appeared from the study of chemical reactors [8]. The study of ODSs become more interesting when the coupling is defined in the boundary conditions. Systems with CBCs can be applied to Lokta-Volterra models, reaction diffusion phenomena and interaction problems, see for example $[9,10,20]$ and references therein. A recent work in this line of research is the one by Asif et al. [7], who study the nonlinear coupled system:

$$
\begin{aligned}
& u^{\prime}(t)=f(t, v(t)), \quad t \in[0,1], \\
& v^{\prime}(t)=g(t, u(t)), \quad t \in[0,1],
\end{aligned}
$$

subject to the nonlinear $\mathrm{CBC}$

$$
h(u(0), v(0), u(1), v(1))=(0,0) .
$$

The authors investigated the existence of at least one solution applying coupled lower and upper solution approach.

Motivated by the works in $[7,11]$, and a wide applications of CBCs, we consider the existence of solution of the following nonlinear coupled system

$$
\begin{array}{ll}
-u^{\prime \prime}(t)=f_{1}(t, v(t)), & t \in[0,1], \\
-v^{\prime \prime}(t)=f_{2}(t, u(t)), & t \in[0,1],
\end{array}
$$

subject to nonlinear CBCs

$$
\begin{aligned}
& \mu\left(u(0), v(0), u^{\prime}(0), v^{\prime}(0), u^{\prime}(1), v^{\prime}(1)\right)=(0,0), \\
& \nu(u(0), v(0))+(u(1), v(1))=(0,0),
\end{aligned}
$$


where $f_{1}, f_{2}:[0,1] \times \mathbf{R} \rightarrow \mathbf{R}, \mu: \mathbf{R}^{6} \rightarrow \mathbf{R}^{2}$ and $\nu: \mathbf{R}^{2} \rightarrow \mathbf{R}^{2}$ are continuous functions.

The results presented in our work are new and having a valuable addition in the existing literature of nonlinear CBCs. The research ideas discussed in $[7,11]$ are extended in our work. In [7] the existence results are established for a first-order nonlinear coupled ODS but in our case the idea is extended for a second-order nonlinear coupled ODS. In [11] the concept of a coupled lower and an upper solution was defined for a single differential equation. On the other hand, in our work that concept is extended for a system of differential equations.

The other interesting aspect of our work is the choice of boundary conditions (BCs) and Definition 2.1. That is (1.7) generalizes most of the usual linear BCs. We mean to say that if $\mu(j, k, l, m, n, o)=(l-$ $n, m-o)$ and $\nu(j, k)=(-j,-k)$, then (1.7) implies the periodic BCs and if $\mu(j, k, l, m, n, o)=(l+n, m+0)$ and $\nu(j, k)=(j, k)$, then (1.7) implies the anti-periodic BCs. Definitely, in order to obtain a solution satisfying these BCs and lying between a subsolution and a supersolution, some additional conditions are required. For example, in the periodic case it suffices that

$$
\begin{gathered}
\alpha_{1}^{\prime}(0) \geq \alpha_{1}^{\prime}(1), \alpha_{2}^{\prime}(0) \geq \alpha_{2}^{\prime}(1), \alpha_{1}(1)=\alpha_{1}(0), \alpha_{2}(1)=\alpha_{2}(0), \\
\left.\beta_{1}^{\prime}(0) \leq \beta_{1}^{\prime}(1), \beta_{2}^{\prime}(0) \leq \beta_{2}^{\prime}(1), \beta_{1}(1)=\beta_{1}(0), \beta_{2}(1)=\beta_{2}(0)\right),
\end{gathered}
$$

and in the anti-periodic case it suffices that

$$
\begin{aligned}
& \alpha_{1}^{\prime}(0) \geq-\beta_{1}^{\prime}(1), \alpha_{2}^{\prime}(0) \geq-\beta_{2}^{\prime}(1),-\alpha_{1}(0)=\beta_{1}(1),-\alpha_{2}(0)=\beta_{2}(1), \\
& \left.\beta_{1}^{\prime}(0) \leq-\alpha_{1}^{\prime}(1), \beta_{2}^{\prime}(0) \leq-\alpha_{2}^{\prime}(1), \alpha_{1}(1)=-\beta_{1}(0), \alpha_{2}(1)=-\beta_{2}(0)\right) .
\end{aligned}
$$

Now the question is that whether the Theorem 2.2 that is designed to study the existence results for a nonlinear coupled differential system with 
nonlinear CBCs is also well equipped to study the existence criterion (1.8)(1.9)? So to tackle this question the concept of a coupled lower and an upper solutions is defined in Definition 2.1 and the results in (1.8)-(1.9) are easily verified by the use of the system of inequalities in (2.1).

Definition 1.1. We say that a function $\left(\alpha_{1}, \alpha_{2}\right) \in C^{2}[0,1] \times C^{2}[0,1]$ is a subsolution of (1.6) if

$$
\begin{array}{ll}
-\alpha_{1}^{\prime \prime}(t) \leq f_{1}\left(t, \alpha_{2}(t)\right), & t \in[0,1], \\
-\alpha_{2}^{\prime \prime}(t) \leq f_{2}\left(t, \alpha_{1}(t)\right), \quad t \in[0,1] .
\end{array}
$$

In the same way, a supersolution is a function $\left(\beta_{1}, \beta_{2}\right) \in C^{2}[0,1] \times$ $C^{2}[0,1]$, that satisfies the same inequalities in reverse order.

For $u, v \in C^{2}[0,1]$, we define the set

$$
[u, v]=\left\{w \in C^{2}[0,1]: u(t) \leq w(t) \leq v(t), t \in[0,1]\right\} .
$$

The following lemma is very useful for our work:

Lemma 1.2. Let $\hat{A}: C^{1}[0,1] \times C^{1}[0,1] \rightarrow C_{0}[0,1] \times C_{0}[0,1] \times \mathbf{R}^{2} \times \mathbf{R}^{2}$ be defined by

$$
\begin{aligned}
{[\hat{A}(u, v)](t)=} & \left(u^{\prime}(t)-u^{\prime}(0)-\varphi \int_{0}^{t} u(s) d s, v^{\prime}(t)-v^{\prime}(0)-\varphi \int_{0}^{t} v(s) d s\right. \\
& \left.(a u(0)+b u(1), c v(0)+d v(1)),\left(a^{\prime} u(0)+b^{\prime} u(1), c^{\prime} v(0)+d^{\prime} v(1)\right)\right)
\end{aligned}
$$

where $\varphi, a, b, c, d, a^{\prime}, b^{\prime}, c^{\prime}$ and $d^{\prime}$ are real constants with $\varphi>0$ such that

$$
(a d-b c)\left(a^{\prime} d^{\prime}-b^{\prime} c^{\prime}\right)\left(e^{-\sqrt{\varphi}}-e^{\sqrt{\varphi}}\right) \neq 0,
$$

and here

$$
C_{0}[0,1]=\left\{x \in C^{2}[0,1]: x(0)=0\right\}
$$


Then $\hat{A}^{-1}$ exists and is continuous and defined by

$$
\begin{aligned}
{\left[\hat{A}^{-1}(y, z, \gamma, \delta, \xi, \zeta)\right] } & =\left(\lambda_{1} e^{\sqrt{\varphi} t}+\lambda_{2} e^{-\sqrt{\varphi} t}+\frac{1}{2} \int_{0}^{t} e^{\sqrt{\varphi}(t-s)} y(s) d s\right. \\
& -\frac{1}{2} \int_{0}^{t} e^{\sqrt{\varphi}(s-t)} y(s) d s, \lambda_{3} e^{\sqrt{\varphi} t}+\lambda_{4} e^{-\sqrt{\varphi} t} \\
& \left.+\frac{1}{2} \int_{0}^{t} e^{\sqrt{\varphi}(t-s)} z(s) d s-\frac{1}{2} \int_{0}^{t} e^{\sqrt{\varphi}(s-t)} z(s) d s\right)
\end{aligned}
$$

with

$$
\begin{aligned}
& \lambda_{1}=\frac{1}{(a d-b c)\left(e^{\sqrt{\varphi}}-e^{-\sqrt{\varphi}}\right)}\left(2 \delta\left(a+b e^{-\sqrt{\varphi}}\right)-d\left(a+b e^{-\sqrt{\varphi}}\right)\right. \\
& \int_{0}^{1} e^{\sqrt{\varphi}(1-s)} y(s) d s+d\left(a+b e^{-\sqrt{\varphi}}\right) \int_{0}^{1} e^{\sqrt{\varphi}(s-1)} y(s) d s \\
& -2 \gamma\left(c+d e^{-\sqrt{\varphi}}\right)+b\left(c+d e^{-\sqrt{\varphi}}\right) \int_{0}^{1} e^{\sqrt{\varphi}(1-s)} y(s) d s \\
& \left.-b\left(c+d e^{-\sqrt{\varphi}}\right) \int_{0}^{1} e^{\sqrt{\varphi}(s-1)} y(s) d s\right) \\
& \lambda_{2}=\frac{1}{(a d-b c)\left(e^{-\sqrt{\varphi}}-e^{\sqrt{\varphi}}\right)}\left(2 \delta\left(a+b e^{\sqrt{\varphi}}\right)-d\left(a+b e^{\sqrt{\varphi}}\right)\right. \\
& \int_{0}^{1} e^{\sqrt{\varphi(1-s)}} y(s) d s+d\left(a+b e^{\sqrt{\varphi}}\right) \int_{0}^{1} e^{\sqrt{\varphi}(s-1)} y(s) d s \\
& -2 \gamma\left(c+d e^{\sqrt{\varphi}}\right)+b\left(c+d e^{\sqrt{\varphi}}\right) \int_{0}^{1} e^{\sqrt{\varphi}(1-s)} y(s) d s \\
& \left.-b\left(c+d e^{\sqrt{\varphi}}\right) \int_{0}^{1} e^{\sqrt{\varphi(s-1)}} y(s) d s\right) \\
& \lambda_{3}=\frac{1}{\left(a^{\prime} d^{\prime}-b^{\prime} c^{\prime}\right)\left(e^{\sqrt{\varphi}}-e^{-\sqrt{\varphi}}\right)}\left(2 \zeta\left(a^{\prime}+b^{\prime} e^{-\sqrt{\varphi}}\right)-d^{\prime}\left(a^{\prime}+b^{\prime} e^{-\sqrt{\varphi}}\right)\right. \\
& \int_{0}^{1} e^{\sqrt{\varphi}(1-s)} z(s) d s+b^{\prime}\left(a^{\prime}+b^{\prime} e^{-\sqrt{\varphi}}\right) \int_{0}^{1} e^{\sqrt{\varphi(s-1)}} z(s) d s \\
& -2 \xi\left(c^{\prime}+d^{\prime} e^{-\sqrt{\varphi}}\right)+b^{\prime}\left(c^{\prime}+d^{\prime} e^{-\sqrt{\varphi}}\right) \int_{0}^{1} e^{\sqrt{\varphi}(1-s)} z(s) d s \\
& \left.-b^{\prime}\left(c^{\prime}+d^{\prime} e^{-\sqrt{\varphi}}\right) \int_{0}^{1} e^{\sqrt{\varphi}(s-1)} z(s) d s\right),
\end{aligned}
$$


and

$$
\begin{aligned}
\lambda_{4}= & \frac{1}{\left(a^{\prime} d^{\prime}-b^{\prime} c^{\prime}\right)\left(e^{-\sqrt{\varphi}}-e^{\sqrt{\varphi}}\right)}\left(2 \zeta\left(a^{\prime}+b^{\prime} e^{\sqrt{\varphi}}\right)-d^{\prime}\left(a^{\prime}+b^{\prime} e^{\sqrt{\varphi}}\right)\right. \\
& \int_{0}^{1} e^{\sqrt{\varphi}(1-s)} z(s) d s+b^{\prime}\left(a^{\prime}+b^{\prime} e^{\sqrt{\varphi}}\right) \int_{0}^{1} e^{\sqrt{\varphi}(s-1)} z(s) d s \\
- & 2 \xi\left(c^{\prime}+d^{\prime} e^{\sqrt{\varphi}}\right)+b^{\prime}\left(c^{\prime}+d^{\prime} e^{\sqrt{\varphi}}\right) \int_{0}^{1} e^{\sqrt{\varphi}(1-s)} z(s) d s \\
- & \left.b^{\prime}\left(c^{\prime}+d^{\prime} e^{\sqrt{\varphi}}\right) \int_{0}^{1} e^{\sqrt{\varphi}(s-1)} z(s) d s\right) .
\end{aligned}
$$

Proof. Choose

$$
\begin{aligned}
& y(t)=u^{\prime}(t)-u^{\prime}(0)-\varphi \int_{0}^{t} u(s) d s \\
& z(t)=v^{\prime}(t)-v^{\prime}(0)-\varphi \int_{0}^{t} v(s) d s,
\end{aligned}
$$

$$
\gamma=a u(0)+b u(1)
$$

$$
\delta=c v(0)+d v(1)
$$

$$
\xi=a^{\prime} u(0)+b^{\prime} u(1)
$$

In the light of (1.12)-(1.17), (1.11) can also be written as

$$
[\hat{A}(u, v)](t)=(y(t), z(t),(\gamma, \delta),(\xi, \zeta))
$$


Differentiating (1.12) with respect to $t$, we have

$$
y^{\prime}(t)=u^{\prime \prime}(t)-\varphi u(t), \quad \varphi>0 .
$$

The general solution of (1.19) can be easily determined using variation of parameters technique alongwith integration by parts and taking limits of integration from 0 to $t$, we have

$$
u(t)=\lambda_{1} e^{\sqrt{\varphi} t}+\lambda_{2} e^{-\sqrt{\varphi} t}+\frac{1}{2} \int_{0}^{t} e^{\sqrt{\varphi}(t-s)} y(s) d s+\frac{1}{2} \int_{0}^{t} e^{\sqrt{\varphi}(s-t)} y(s) d s .
$$

$\lambda_{1}$ and $\lambda_{2}$ can be easily determined with the help of (1.14) and (1.15) as

$$
\begin{gathered}
\gamma=\left(a+b e^{\sqrt{\varphi}}\right) \lambda_{1}+\left(a+b e^{-\sqrt{\varphi}}\right) \lambda_{2}+\frac{b}{2}\left(\int_{0}^{1} e^{\sqrt{\varphi}(1-s)} y(s) d s+e^{\sqrt{\varphi}(s-1)} y(s) d s\right) \\
\delta=\left(c+d e^{\sqrt{\varphi}}\right) \lambda_{1}+\left(c+d e^{-\sqrt{\varphi}}\right) \lambda_{2}+\frac{d}{2}\left(\int_{0}^{1} e^{\sqrt{\varphi}(1-s)} y(s) d s+e^{\sqrt{\varphi}(s-1)} y(s) d s\right) .
\end{gathered}
$$

Solving the system of equations (1.21), we have

$$
\begin{aligned}
& \lambda_{1}=\frac{1}{(a d-b c)\left(e^{\sqrt{\varphi}}-e^{-\sqrt{\varphi}}\right)}\left(2 \delta\left(a+b e^{-\sqrt{\varphi}}\right)-d\left(a+b e^{-\sqrt{\varphi}}\right)\right. \\
& \int_{0}^{1} e^{\sqrt{\varphi}(1-s)} y(s) d s+d\left(a+b e^{-\sqrt{\varphi}}\right) \int_{0}^{1} e^{\sqrt{\varphi}(s-1)} y(s) d s \\
& -2 \gamma\left(c+d e^{-\sqrt{\varphi}}\right)+b\left(c+d e^{-\sqrt{\varphi}}\right) \int_{0}^{1} e^{\sqrt{\varphi}(1-s)} y(s) d s \\
& \left.-\mathrm{b}\left(c+d e^{-\sqrt{\varphi}}\right) \int_{0}^{1} e^{\sqrt{\varphi}(s-1)} y(s) d s\right) \\
& \lambda_{2}=\frac{1}{(a d-b c)\left(e^{-\sqrt{\varphi}}-e^{\sqrt{\varphi}}\right)}\left(2 \delta\left(a+b e^{\sqrt{\varphi}}\right)-d\left(a+b e^{\sqrt{\varphi}}\right)\right. \\
& \int_{0}^{1} e^{\sqrt{\varphi}(1-s)} y(s) d s+d\left(a+b e^{\sqrt{\varphi}}\right) \int_{0}^{1} e^{\sqrt{\varphi}(s-1)} y(s) d s
\end{aligned}
$$


Coupled lower and upper solution approach for the existence of ... 107

$$
\begin{aligned}
& -2 \gamma\left(c+d e^{\sqrt{\varphi}}\right)+b\left(c+d e^{\sqrt{\varphi}}\right) \int_{0}^{1} e^{\sqrt{\varphi}(1-s)} y(s) d s \\
& \left.-\mathrm{b}\left(c+d e^{\sqrt{\varphi}}\right) \int_{0}^{1} e^{\sqrt{\varphi}(s-1)} y(s) d s\right) .
\end{aligned}
$$

Similarly on the same line, it can be easily shown that

$$
v(t)=\lambda_{3} e^{\sqrt{\varphi} t}+\lambda_{4} e^{-\sqrt{\varphi} t}+\frac{1}{2} \int_{0}^{t} e^{\sqrt{\varphi}(t-s)} z(s) d s+\frac{1}{2} \int_{0}^{t} e^{\sqrt{\varphi}(s-t)} z(s) d s,
$$

with

$$
\begin{aligned}
\lambda_{3}= & \frac{1}{\left(a^{\prime} d^{\prime}-b^{\prime} c^{\prime}\right)\left(e^{\sqrt{\varphi}}-e^{-\sqrt{\varphi}}\right)}\left(2 \zeta\left(a^{\prime}+b^{\prime} e^{-\sqrt{\varphi}}\right)-d^{\prime}\left(a^{\prime}+b^{\prime} e^{-\sqrt{\varphi}}\right)\right. \\
& \int_{0}^{1} e^{\sqrt{\varphi}(1-s)} z(s) d s+b^{\prime}\left(a^{\prime}+b^{\prime} e^{-\sqrt{\varphi}}\right) \int_{0}^{1} e^{\sqrt{\varphi}(s-1)} z(s) d s \\
- & 2 \xi\left(c^{\prime}+d^{\prime} e^{-\sqrt{\varphi}}\right)+b^{\prime}\left(c^{\prime}+d^{\prime} e^{-\sqrt{\varphi}}\right) \int_{0}^{1} e^{\sqrt{\varphi}(1-s)} z(s) d s \\
- & \left.b^{\prime}\left(c^{\prime}+d^{\prime} e^{-\sqrt{\varphi}}\right) \int_{0}^{1} e^{\sqrt{\varphi}(s-1)} z(s) d s\right)
\end{aligned}
$$

and

$$
\begin{aligned}
\lambda_{4}= & \frac{1}{\left(a^{\prime} d^{\prime}-b^{\prime} c^{\prime}\right)\left(e^{-\sqrt{\varphi}}-e^{\sqrt{\varphi}}\right)}\left(2 \zeta\left(a^{\prime}+b^{\prime} e^{\sqrt{\varphi}}\right)-d^{\prime}\left(a^{\prime}+b^{\prime} e^{\sqrt{\varphi}}\right)\right. \\
& \int_{0}^{1} e^{\sqrt{\varphi}(1-s)} z(s) d s+b^{\prime}\left(a^{\prime}+b^{\prime} e^{\sqrt{\varphi}}\right) \int_{0}^{1} e^{\sqrt{\varphi}(s-1)} z(s) d s
\end{aligned}
$$




$$
\begin{aligned}
& -2 \xi\left(c^{\prime}+d^{\prime} e^{\sqrt{\varphi}}\right)+b^{\prime}\left(c^{\prime}+d^{\prime} e^{\sqrt{\varphi}}\right) \int_{0}^{1} e^{\sqrt{\varphi}(1-s)} z(s) d s \\
(1.25) & \left.-b^{\prime}\left(c^{\prime}+d^{\prime} e^{\sqrt{\varphi}}\right) \int_{0}^{1} e^{\sqrt{\varphi(s-1)}} z(s) d s\right) .
\end{aligned}
$$

(1.18) can also be written as

$$
(u(t), v(t))=\left[\hat{A}^{-1}(y(t), z(t),(\gamma, \delta),(\xi, \zeta))\right] .
$$

Hence (1.20)-(1.26) prove the required result.

\section{Coupled lower and upper solutions}

The following definition is very useful to construct the statement of the main result, and also it covers different possibilities for the nonlinear boundary functions $\mu$ and $\nu$.

Definition 2.1. We say that $\left(\alpha_{1}, \alpha_{2}\right),\left(\beta_{1}, \beta_{2}\right) \in C^{2}[0,1] \times C^{2}[0,1]$ are coupled lower and upper solutions for the problem (1.6) and (1.7) if $\left(\alpha_{1}, \alpha_{2}\right)$ is a subsolution and $\left(\beta_{1}, \beta_{2}\right)$ a supersolution for the system (1.6), $\left(\alpha_{1}, \alpha_{2}\right) \preceq$ $\left(\beta_{1}, \beta_{2}\right)$, if $\alpha_{1} \leq \beta_{1}$ and $\alpha_{2} \leq \beta_{2}$, and

$$
\begin{gathered}
\mu\left(\beta_{1}(0), \beta_{2}(0), \beta_{1}^{\prime}(0), \beta_{2}^{\prime}(0), \beta_{1}^{\prime}(1), \beta_{2}^{\prime}(1)\right) \preceq(0,0) \\
\preceq \mu\left(\alpha_{1}(0), \alpha_{2}(0), \alpha_{1}^{\prime}(0), \alpha_{2}^{\prime}(0), \alpha_{1}^{\prime}(1), \alpha_{2}^{\prime}(1)\right), \\
\mu\left(\beta_{1}(0), \beta_{2}(0), \beta_{1}^{\prime}(0), \beta_{2}^{\prime}(0), \alpha_{1}^{\prime}(1), \alpha_{2}^{\prime}(1)\right) \preceq(0,0) \\
\preceq \mu\left(\alpha_{1}(0), \alpha_{2}(0), \alpha_{1}^{\prime}(0), \alpha_{2}^{\prime}(0), \beta_{1}^{\prime}(1), \beta_{2}^{\prime}(1)\right), \\
\quad\left(\alpha_{1}(1), \alpha_{2}(1)\right)+\nu\left(\beta_{1}(0), \beta_{2}(0)\right)=(0,0), \\
\quad\left(\beta_{1}(1), \beta_{2}(1)\right)+\nu\left(\alpha_{1}(0), \alpha_{2}(0)\right)=(0,0), \\
\left(\alpha_{1}(1), \alpha_{2}(1)\right)+\nu\left(\alpha_{1}(0), \alpha_{2}(0)\right)=(0,0), \\
\left(\beta_{1}(1), \beta_{2}(1)\right)+\nu\left(\beta_{1}(0), \beta_{2}(0)\right)=(0,0) .
\end{gathered}
$$


Theorem 2.2. Assume that $\left(\alpha_{1}, \alpha_{2}\right),\left(\beta_{1}, \beta_{2}\right)$ are coupled lower and upper solutions for the problem (1.6)-(1.7). Suppose that $\mu$ is nondecreasing in the third and fourth arguments. In addition suppose that the function $\nu$ in $\left[\alpha_{1}(0), \beta_{1}(0)\right] \times\left[\alpha_{2}(0), \beta_{2}(0)\right]$ is monotone and the functions

$$
\begin{aligned}
& \mu_{\left(\alpha_{1}, \alpha_{2}\right)}(x, y):=\mu\left(\alpha_{1}(0), \alpha_{2}(0), \alpha_{1}^{\prime}(0), \alpha_{2}^{\prime}(0), x, y\right), \\
& \mu_{\left(\beta_{1}, \beta_{2}\right)}(x, y):=\mu\left(\beta_{1}(0), \beta_{2}(0), \beta_{1}^{\prime}(0), \beta_{2}^{\prime}(0), x, y\right),
\end{aligned}
$$

have got the same kind of monotonocity as $\nu$, then there exists at least one solution $(u, v) \in\left[\alpha_{1}, \beta_{1}\right] \times\left[\alpha_{2}, \beta_{2}\right]$ of the problem (1.6)-(1.7).

Proof. Let $\varphi>0$ and consider the modified system

$$
\begin{aligned}
& -u^{\prime \prime}(t)+\varphi u(t)=F_{1}^{*}(t, u(t), v(t)), \quad t \in[0,1], \\
& -v^{\prime \prime}(t)+\varphi v(t)=F_{2}^{*}(t, u(t), v(t)), \quad t \in[0,1], \\
& \mu^{*}\left(u(0), v(0), u(1), v(1), u^{\prime}(0), v^{\prime}(0)\right)=(u(0), v(0)), \\
& (u(1), v(1))+\nu^{*}(u(0), v(0))=(0,0),
\end{aligned}
$$

with

$$
F_{1}^{*}(t, u(t), v(t))= \begin{cases}f_{1}\left(t, \beta_{2}(t)\right)+\varphi \beta_{1}(t) & \text { if } v(t)>\beta_{2}(t), u(t)>\beta_{1}(t), \\ f_{1}(t, v(t))+\varphi \beta_{1}(t) & \text { if } \alpha_{2}(t) \leq v(t) \leq \beta_{2}(t), u(t)>\beta_{1}(t), \\ f_{1}\left(t, \alpha_{2}(t)\right)+\varphi \beta_{1}(t) & \text { if } v(t)<\alpha_{2}(t), u(t)>\beta_{1}(t), \\ f_{1}\left(t, \beta_{2}(t)\right)+\varphi u(t) & \text { if } v(t)>\beta_{2}(t), \alpha_{1}(t) \leq u(t) \leq \beta_{1}(t), \\ f_{1}(t, v(t))+\varphi u(t) & \text { if } \alpha_{2}(t) \leq v(t) \leq \beta_{2}(t), \\ & \alpha_{1}(t) \leq u(t) \leq \beta_{1}(t), \\ f_{1}\left(t, \alpha_{2}(t)\right)+\varphi u(t) & \text { if } v(t)<\alpha_{2}(t), \alpha_{1}(t) \leq u(t) \leq \beta_{1}(t), \\ f_{1}\left(t, \beta_{2}(t)\right)+\varphi \alpha_{1}(t) & \text { if } v(t)>\beta_{2}(t), u(t)<\alpha_{1}(t), \\ f_{1}(t, v(t))+\varphi \alpha_{1}(t) & \text { if } \alpha_{2}(t) \leq v(t) \leq \beta_{2}(t), u(t)<\alpha_{1}(t), \\ f_{1}\left(t, \alpha_{2}(t)\right)+\varphi \alpha_{1}(t) & \text { if } v(t)<\alpha_{2}(t), u(t)<\alpha_{1}(t),\end{cases}
$$

and 


$$
\begin{gathered}
F_{2}^{*}(t, u(t), v(t))= \begin{cases}f_{2}\left(t, \beta_{1}(t)\right)+\varphi \beta_{2}(t) & \text { if } v(t)>\beta_{2}(t), u(t)>\beta_{1}(t), \\
f_{2}(t, u(t))+\varphi \beta_{2}(t) & \text { if } \alpha_{1}(t) \leq u(t) \leq \beta_{1}(t), v(t)>\beta_{2}(t), \\
f_{2}\left(t, \alpha_{1}(t)\right)+\varphi \beta_{2}(t) & \text { if } u(t)<\alpha_{1}(t), v(t)>\beta_{2}(t), \\
f_{2}\left(t, \beta_{1}(t)\right)+\varphi v(t) & \text { if } u(t)>\beta_{1}(t), \alpha_{2}(t) \leq v(t) \leq \beta_{2}(t), \\
f_{2}(t, u(t))+\varphi v(t) & \text { if } \alpha_{1}(t) \leq u(t) \leq \beta_{1}(t), \\
f_{2}\left(t, \alpha_{1}(t)\right)+\varphi v(t) & \text { if } u(t)<\alpha_{1}(t), \alpha_{2}(t) \leq v(t) \leq \beta_{2}(t), \\
f_{2}\left(t, \beta_{1}(t)\right)+\varphi \alpha_{2}(t) & \text { if } u(t)>\beta_{1}(t), v(t)<\alpha_{2}(t), \\
f_{2}(t, u(t))+\varphi \alpha_{2}(t) & \text { if } \alpha_{1}(t) \leq u(t) \leq \beta_{1}(t), v(t)<\alpha_{2}(t), \\
f_{2}\left(t, \alpha_{1}(t)\right)+\varphi \alpha_{2}(t) \quad \text { if } u(t)<\alpha_{1}(t), v(t)<\alpha_{2}(t),\end{cases} \\
\mu^{*}(j, k, l, m, n, o)=\phi(0,(j, k)+\mu(j, k, l, m, n, o)), \\
\nu^{*}(j, k)=\nu(\phi(0,(j, k))), \\
\phi(t,(x, y))= \begin{cases}\left(\beta_{1}(t), \beta_{2}(t)\right) & \text { if }(x, y) \npreceq\left(\beta_{1}, \beta_{2}\right) \\
(x, y) & \text { if }\left(\alpha_{1}, \alpha_{2}\right) \preceq(x, y) \preceq\left(\beta_{1}, \beta_{2}\right) \\
\left(\alpha_{1}(t), \alpha_{2}(t)\right) & \text { if }(x, y) \nsucceq\left(\alpha_{1}, \alpha_{2}\right),\end{cases}
\end{gathered}
$$

Note that if $(u, v) \in\left[\alpha_{1}, \beta_{1}\right] \times\left[\alpha_{2}, \beta_{2}\right]$ is a solution of $(2.2)$, then $(u, v)$ is a solution of (1.6)-(1.7).

For the sake of simplicity we divide the proof in three steps:

\section{Step 1:}

We define the mappings

$$
\hat{A}, \hat{B}: C^{1}[0,1] \times C^{1}[0,1] \rightarrow C_{0}[0,1] \times C_{0}[0,1] \times \mathbf{R}^{2} \times \mathbf{R}^{2},
$$

by

$$
\begin{aligned}
{[\hat{A}(u, v)](t)=} & \left(u^{\prime}(t)-u^{\prime}(0)-\varphi \int_{0}^{t} u(s) d s, v^{\prime}(t)-v^{\prime}(0)-\varphi \int_{0}^{t} v(s) d s\right. \\
& (u(0), v(0)),(u(1), v(1)))
\end{aligned}
$$

and

$$
[\hat{B}(u, v)](t)=\left(\int_{0}^{t} F_{1}^{*}(s, u(s), v(s)) d s, \int_{0}^{t} F_{2}^{*}(s, u(s), v(s)) d s\right.
$$




$$
\begin{aligned}
& \mu^{*}\left(u(0), v(0), u(1), v(1), u^{\prime}(0), v^{\prime}(0)\right), \\
& \left.-\nu^{*}(u(0), v(0))\right)
\end{aligned}
$$

Clearly $\hat{B}$ is continuous and compact by the direct application of ArzelaAscoli theorem. Also from Lemma 1.2 with $a=1, b=0, c=1, d=0$ and $a^{\prime}=0, b^{\prime}=1, c^{\prime}=0, d^{\prime}=1, \hat{A}^{-1}$, exists and is continuous.

On the other hand, solving (2.2) is equivalent to find a fixed point of

$$
\hat{A}^{-1} \hat{B}: C^{1}[0,1] \times C^{1}[0,1] \rightarrow C^{1}[0,1] \times C^{1}[0,1] .
$$

Now, Schauder's fixed point theorem guarantees the existence of at least a fixed point since $\hat{A}^{-1} \hat{B}$ is continuous and compact.

Step 2: If $(u, v)$ is a solution of $(2.2)$, then $(u, v) \in\left[\alpha_{1}, \beta_{1}\right] \times\left[\alpha_{2}, \beta_{2}\right]$. By definition of $\mu^{*}$, we see that $(u(0), v(0)) \in\left[\alpha_{1}(0), \beta_{1}(0)\right] \times\left[\alpha_{2}(0), \beta_{2}(0)\right]$. Thus, if $\nu$ is nondecreasing, we have by condition $(2.1)$

$$
\begin{aligned}
& \left(\alpha_{1}(1), \alpha_{2}(1)\right)=-\nu\left(\beta_{1}(0), \beta_{2}(0)\right) \preceq-\nu(u(0), v(0)) \\
& =(u(1), v(1)) \preceq-\nu\left(\alpha_{1}(0), \alpha_{2}(0)\right) \\
& \left(\alpha_{1}(1), \alpha_{2}(1)\right) \preceq(u(1), v(1)) \preceq\left(\beta_{1}(1), \beta_{2}(1)\right),
\end{aligned}
$$

similarly, if $\nu$ is nonincreasing, then (2.3) holds. Hence $(u(1), v(1)) \in$ $\left[\alpha_{1}(1), \beta_{1}(1)\right] \times\left[\alpha_{2}(1), \beta_{2}(1)\right]$. Now, it remains to show that $(u, v) \in\left[\alpha_{1}, \beta_{1}\right] \times$ $\left[\alpha_{2}, \beta_{2}\right]$ for $t \in(0,1)$.

We claim $(u, v) \preceq\left(\beta_{1}, \beta_{2}\right)$. If $(u, v) \npreceq\left(\beta_{1}, \beta_{2}\right)$, then $u \npreceq \beta_{1}$ and/or $v \npreceq$ $\beta_{2}$. If $u \npreceq \beta_{1}$, then there exist some $t_{0} \in[0,1]$ such that $u\left(t_{0}\right)-\beta_{1}\left(t_{0}\right)>0$. So, $u-\beta_{1}$ attains a positive maximum at $t_{0} \in[0,1]$. Thus $\left(u-\beta_{1}\right)^{\prime}\left(t_{0}\right)=0$ and $\left(u-\beta_{1}\right)^{\prime \prime}\left(t_{0}\right)<0$. But

$$
\begin{gathered}
\left.u-\beta_{1}\right)^{\prime \prime}\left(t_{0}\right)>-F_{1}^{*}\left(t_{0}, u\left(t_{0}\right), v\left(t_{0}\right)\right)+\varphi u\left(t_{0}\right)+f_{1}\left(t_{0}, \beta_{2}\left(t_{0}\right)\right) \\
=-f_{1}\left(t_{0}, \beta_{2}\left(t_{0}\right)\right)-\varphi \beta_{1}\left(t_{0}\right)+\varphi u\left(t_{0}\right)+f_{1}\left(t_{0}, \beta_{2}\left(t_{0}\right)\right) \\
=\varphi\left(u\left(t_{0}\right)-\beta_{1}\left(t_{0}\right)\right)>0,
\end{gathered}
$$

a contradiction. Similarly one can show that $\left(\alpha_{1}, \alpha_{2}\right) \preceq(u, v)$. Hence $(u, v) \in\left[\alpha_{1}, \beta_{1}\right] \times\left[\alpha_{2}, \beta_{2}\right]$.

Step 3: If $(u, v)$ is a solution of $(2.2)$ then $(u, v)$ satisfies (1.7). 
We claim

$$
\begin{aligned}
& \left(\alpha_{1}(0), \alpha_{2}(0)\right) \preceq(u(0), v(0))+\mu\left(u(0), v(0), u^{\prime}(0), v^{\prime}(0), u^{\prime}(1), v^{\prime}(1)\right) \\
& \preceq\left(\beta_{1}(0), \beta_{2}(0)\right) .
\end{aligned}
$$
then

$$
\begin{aligned}
& (u(0), v(0))=\mu^{*}\left(u(0), v(0), u^{\prime}(0), v^{\prime}(0), u^{\prime}(1), v^{\prime}(1)\right) \\
& =\phi\left(0,(u(0), v(0))+\mu\left(u(0), v(0), u^{\prime}(0), v^{\prime}(0), u^{\prime}(1), v^{\prime}(1)\right)\right) \\
& =\left(\alpha_{1}(0), \alpha_{2}(0)\right) .
\end{aligned}
$$

If $\nu$ is nondecreasing, we have

$$
(u(1), v(1))=-\nu(u(0), v(0))=-\nu\left(\alpha_{1}(0), \alpha_{2}(0)\right)=\left(\beta_{1}(1), \beta_{2}(1)\right) .
$$

Using (2.5), (2.6) and Step 2, we have $\left(u^{\prime}(0), v^{\prime}(0)\right) \succeq\left(\alpha_{1}^{\prime}(0), \alpha_{2}^{\prime}(0)\right)$ and $\left(u^{\prime}(1), v^{\prime}(1)\right) \succeq\left(\beta_{1}^{\prime}(1), \beta_{2}^{\prime}(1)\right)$. But

$$
\begin{aligned}
(u(0), v(0)) & +\mu\left(u(0), v(0), u^{\prime}(0), v^{\prime}(0), u^{\prime}(1), v^{\prime}(1)\right) \\
& =\left(\alpha_{1}(0), \alpha_{2}(0)\right)+\mu\left(\alpha_{1}(0), \alpha_{2}(0), u^{\prime}(0), v^{\prime}(0), u^{\prime}(1), v^{\prime}(1)\right) \\
& \succeq\left(\alpha_{1}(0), \alpha_{2}(0)\right)+\mu\left(\alpha_{1}(0), \alpha_{2}(0), \alpha_{1}^{\prime}(0), \alpha_{2}^{\prime}(0), u^{\prime}(1), v^{\prime}(1)\right) \\
& =\left(\alpha_{1}(0), \alpha_{2}(0)\right)+\mu_{\left(\alpha_{1}, \alpha_{2}\right)}\left(u^{\prime}(1), v^{\prime}(1)\right) \\
& \succeq\left(\alpha_{1}(0), \alpha_{2}(0)\right)+\mu\left(\alpha_{1}(0), \alpha_{2}(0), \alpha_{1}^{\prime}(0), \alpha_{2}^{\prime}(0), \beta_{1}^{\prime}(1), \beta_{2}^{\prime}(1)\right) \\
& \succeq\left(\alpha_{1}(0), \alpha_{2}(0)\right),
\end{aligned}
$$


a contradiction. Similarly if $\nu$ is nonincreasing we get same contradiction. Consequently, 2.4 holds. By definition of $\nu^{*}$ and Step 2, the second boundary condition is obvious. Consequently $(u, v)$ satisfies (1.7). Hence the system of BVPs (1.6)-(1.7) has a solution $(u, v) \in\left[\alpha_{1}, \beta_{1}\right] \times\left[\alpha_{2}, \beta_{2}\right]$.

\section{Examples}

Example 3.1. Consider the nonlinear coupled boundary value system

$$
\begin{gathered}
-u^{\prime \prime}(t)=v^{5}(t)-20 \sqrt{\sin (t)}, \quad t \in[0,1], \\
-v^{\prime \prime}(t)=u^{5}(t)-16 \sqrt{\cos (t-1)}, \quad t \in[0,1],
\end{gathered}
$$

with the following nonlinear CBCs

$$
\begin{gathered}
\left(u(0) u^{\prime}(0)-v(0) v^{\prime}(0), u(0) u^{\prime}(1)-v^{\prime}(0) v^{\prime}(1)\right)=(0,0), \\
(\sqrt{\tan (u(0)) u(1)+\tan (v(0)) v(1)}, \sqrt{\tan (u(0))+\tan (v(0)) v(1)})=(0,0) .
\end{gathered}
$$

Let $\alpha_{1}(t)=-\frac{t^{5}}{4}, \alpha_{2}(t)=-\frac{t^{7}}{5}$ and $\beta_{1}(t)=\frac{t^{5}}{4}, \beta_{2}(t)=\frac{t^{7}}{5}$. It is easy to show that $\left(\alpha_{1}, \alpha_{2}\right),\left(\beta_{1}, \beta_{2}\right)$ are a subsolution and a supersolution of the system (3.1), respectively. Further, $\left(\alpha_{1}, \alpha_{2}\right)$ and $\left(\beta_{1}, \beta_{2}\right)$ satisfy the system (2.1). Hence by Theorem 2.2, the system of BVPs (3.1)-(3.2) has at least one solution $(u, v) \in\left[\alpha_{1}, \beta_{1}\right] \times\left[\alpha_{2}, \beta_{2}\right]$.

Example 3.2. Consider the nonlinear coupled boundary value system

$$
\begin{gathered}
-u^{\prime \prime}(t)=t^{2}+\sin (v(t)), \quad t \in[0,1], \\
-v^{\prime \prime}(t)=5 t^{2}+\cos (u(t)+4)+\sin (u(t)-4), \quad t \in[0,1],
\end{gathered}
$$

with the following nonlinear CBCs

$$
\left(u(0) u^{\prime}(1)-v^{\prime}(0) v^{\prime}(1), u^{\prime}(0) u^{\prime}(1)-v^{\prime}(1)\right)=(0,0),
$$




$$
(\sin (u(0))+u(0) v(1), \sin (v(0))+v(0) u(1))=(0,0) .
$$

Let $\alpha_{1}(t)=t^{2}, \alpha_{2}(t)=t^{3}$ and $\beta_{1}(t)=t, \beta_{2}(t)=t$. It is easy to show that $\left(\alpha_{1}, \alpha_{2}\right),\left(\beta_{1}, \beta_{2}\right)$ are a subsolution and a supersolution of the system (3.3), respectively. Further, $\left(\alpha_{1}, \alpha_{2}\right)$ and $\left(\beta_{1}, \beta_{2}\right)$ satisfy the system (2.1). Hence by Theorem 2.2, the system of BVPs (3.3)-(3.4) has at least one solution $(u, v) \in\left[\alpha_{1}, \beta_{1}\right] \times\left[\alpha_{2}, \beta_{2}\right]$.

\section{Conclusion}

In this article, coupled lower and upper solution methodology is used to investigate the existence of solution of second-order nonlinear coupled system with nonlinear CBCs. The ideas presented in [7, 11] are extended in our article. Also it is worth mentioning that the BCs (1.7) generalize most of the BCs like periodic and anti-periodic BCs. Moreover to verify the classical results (1.8)-(1.9), the concept of coupled lower and upper solutions is defined in Section 2. Some examples are taken to ensure the validity of the theoretical results.

\section{Conflict of interests}

The authors declare that there is no conflict of interests regarding the publication of the paper.

\section{Acknowledgment}

The authors want to thank the anonymous referees for their valuable suggestions that improved the quality of the article. They owe thanks to Ms Saria Kazmi who is serving in Virtual University of Pakistan as an instructor. Her much needed assistance as a proofreader to make this research paper syntactically and linguistically correct is really commendable.

\section{References}


[1] N.A. Asif, R. A. Khan; Positive solutions to singular system with fourpoint coupled boundary conditions, J. Math. Anal. Appl., 386, no. 2, pp. 848-861, (2012).

[2] N.A. Asif, P.W. Eloe, R. A. Khan; Positive solutions for a system of singular second order nonlocal boundary value problems, J. Korean Math. Soc., 47, no. 5, 985-1000, (2010).

[3] L. Hu, L. Wang; Multiple positive solutions of boundary value problems for systems of nonlinear second-order differential equations, J. Math. Anal. Appl., 335, no. 2, pp. 1052-1060, (2007).

[4] Y. Zhou, Y. Xu; Positive solutions of three-point boundary value problems for systems of nonlinear second order ordinary differential equations, J. Math. Anal. Appl., 320, no. 2, pp. 578-590, (2006).

[5] J. Yang, Z. Wei; On existence of positive solutions of Sturm-Liouville boundary value problems for a nonlinear singular differential system, J. Appl. Math. Comput., 217, pp. 6097-6104, (2011).

[6] G. Infante, F. M. Minhós, P. Pietramala; Non-negative solutions of systems of ODES with coupled boundary conditions, Communications in nonlinear science and numerical simulations, 17, pp. 4952-4960, (2012).

[7] N. A. Asif, I. Talib, C. Tunc; Existence of solution for first-order coupled system with nonlinear coupled boundary conditions, Bound. Val. Probl., pp. 9, (2015).

[8] R. Aris; Introduction to the analysis of chemical reactors, PrenticeHall, Englewood Cliffs, NJ, (1965).

[9] H. Amann; Parabolic evolution equations with nonlinear boundary conditions. Part 1 (Berkeley, Calif., 1983), 17-27, Proc. Sympos. Pure Math., 45, Part 1, Amer. Math. Soc., Providence, RI, (1986).

[10] F. A. Mehmeti, S. Nicaise; Nonlinear interaction problems, Nonlinear Anal., 20, no. 1, pp. 27-61, (1993).

[11] D. Franco, D. O'Regan; Existence of solutions to second order problems with nonlinear boundary conditions, Dynamical systems and differential equations (Wilmington, NC, 2002). Discrete Contin. Dyn. Syst., suppl., pp. 273-280, (2003). 
[12] L. K. Jackson; Subfunctions and second-order ordinary differential inequalities, Advances in Math., 2, pp. 307-363, (1968).

[13] O. Perron; Oskar Eine neue Behandlung der ersten Randwertaufgabe für $\Delta u=0$, (German) Math. Z., 18, no. 1, pp. 42-54, (1923).

[14] K. Schmitt; A nonlinear boundary value problem, J. Differential Equations, 7, pp. 527-537, (1970).

[15] Y. Zhang; Positive solutions of singular sublinear Dirichlet boundary value problems, SIAM J. Math. Anal., 26, no. 2, pp. 329-339, (1995).

[16] Y. Zhang; The existence of solutions to nonlinear second order periodic boundary value problems, Nonlinear Anal., 76, pp. 140-152, (2013).

[17] H. Amann; Parabolic evolution and nonlinear boundary conditions, J. Differential Equations, 72, pp. 201-269, (1988).

[18] D.G. Aronson; A comparison method for stability analysis of nonlinear parabolic problems, SIAM Rev., 20, pp. 245-264, (1978).

[19] A. Zettle; Sturm-Liouville Theory, Math. Surveys Monogr., Vol. 121, Amer. Math. Soc., Providence, RI, (2005).

[20] A. Leung; A semilinear reaction-diffusion prey-predator system with nonlinear coupled boundary conditions: Equilibrium and stability, Indiana Univ. Math. J., 31, pp. 223-241, (1982).

[21] S. Agmon, A. Douglis, L. Nirenberg; Estimates near the boundary for solutions of elliptic partial differential equations satisfying general boundary conditions II, Commun. Pure Appl. Math., 17, pp. 35-92, (1964).

[22] S. Cardanobile, D. Mugnolo; Parabolic systems with coupled boundary conditions, J. Differ. Equ., 247, pp. 1229-1248, (2009).

[23] A. Wang, J. Sun, A. Zettl; The classification of self-adjoint boundary conditions: separated, coupled, J. Funct. Anal., 255, pp. 1554-1573, (2008).

[24] G. Infante, P. Pietramala; Multiple nonnegative solutions of systems with coupled nonlinear boundary conditions, Math. Meth. Appl. Sci., 37, pp. 20080-2090, (2014). 
[25] I. Talib, N.A. Asif, C. Tunc; Existence of solutions to second-order nonlinear coupled systems with nonlinear coupled boundary conditions, Electron. J. Diff. Equ., 2015, no. 313, pp. 11, (2014).

\section{Imran Talib}

School of Science,

Department of Mathematics,

University of Management and Technology,

CII Johar Town, Lahore,

Pakistan

e-mail : imrantaalib@gmail.com

\section{Naseer Ahmad Asif}

School of Science,

Department of Mathematics,

University of Management and Technology,

CII Johar Town, Lahore,

Pakistan

e-mail : naseerasif@yahoo.com

and

Cemil Tunc

Department of Mathematics

Faculty of Sciences,

Yuzuncu Yil University,

Van,

Turkey

e-mail : cemtunc@yahoo.com 